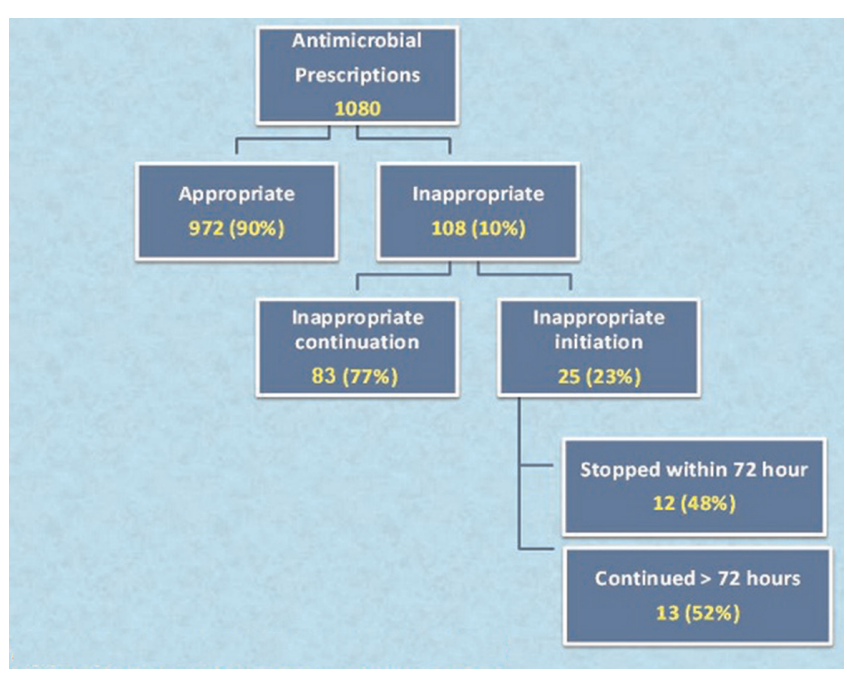

Abstract P0-0549 Figure 2 Appropriateness of Antimicrobial use

\begin{tabular}{lll} 
Abstract PO-0549 Table 2 & & \\
\hline Overall & $\begin{array}{l}\text { Inappropriate (\%) } \\
10 \%\end{array}$ & p-value \\
Location & $12 \%$ & \\
NICU & $5 \%$ & 0.002 \\
ICN & & \\
Indications & $7 \%$ & \\
Perinatal Infection & $12 \%$ & $<0.001$ \\
Healthcare associated Infection & $41 \%$ & \\
Surgical Prophylaxis & $0 \%$ & \\
Medical Prophylaxis & &
\end{tabular}

\section{PO-0550 USE OF PROFILATIC ANTIBIOTICS FOR PRETERM INFANTS-}

FE Martinez', 'WAG Ferri, 'MM Mussi-Pinhata, ${ }^{2}$ VOS Abdallah, CR Leone ${ }^{3},{ }^{3}$ BNNR Brazilian Network on Neonatl Research. 'Pediatrics, University of Sao Paulo, Ribeirao Preto, Brazil; ${ }^{2}$ Pediatrics, Federal University of Uberlandia, Uberlandia, Brazil, ${ }^{3}$ Pediatrics, University of Sao Paulo, Saõ Paulo, Brazil

\subsection{6/archdischild-2014-307384.1192}

The objective was to explore the association between antibiotic use in the first $72 \mathrm{~h}$ of life in low risk for infection preterm infants and nutritional performance.

Among the 4344 children born between 2010 and 2012 in 16 centres of the BNNR weighing $<1495$ g, 2395 infants presented no maternal history of chorioamnionitis, $<18 \mathrm{~h}$ of ruptured membranes, no diagnosis of sepsis in the first $72 \mathrm{~h}$ of life and no congenital malformations. Antibiotics were given to 959 infants during the first $72 \mathrm{~h}$ of life and 1436 received no antibiotics.

The use of antibiotic was more frequent among children with worst birth conditions and those infants presented poorer evolution parameters except for lower incidence of late onset sepsis.

In nutritional terms, after binary logistic regression (BLR), the use of antibiotics remained as independent risk factors to take over 14 days to regain birth weight and for having lost more than $1 \mathrm{z}$-score in weight at discharge.

Centres were stratified by the percentage of antibiotic use. Prenatal and birth condition of the two centres strata were very similar. Infants from Conservative centres presented better nutritional performance, but higher incidence of late onset sepsis, use of antibiotics after $72 \mathrm{hs,} \mathrm{more} \mathrm{days} \mathrm{of} \mathrm{oxygen} \mathrm{use.}$

At BLR for use of oxygen at $36 \mathrm{CA}$, neither centre strata, nor use of antibiotics during the first 72 hs of life maintained the association found on the univariate analysis.

The use of antibiotics in the first $72 \mathrm{~h}$ was independently associated with worse nutritional performance.

\section{PO-0551 AN AUDIT OF ANTIBIOTIC USE FOR EARLY ONSET NEONATAL INFECTION IN A NORTHERN IRISH DISTRICT GENERAL HOSPITAL}

L McDowell, N Geoghegan, M Hogan, J Henderson. Paediatrics, Craigavon Area Hospital, Portadown, UK

\subsection{6/archdischild-2014-307384.1193}

Background Early onset neonatal sepsis is a significant cause of morbidity and mortality. The decision to treat has consequences and a balance must be reached between identifying sick babies and avoiding the potential hazards associated with investigations and over-treatment. Using the 2012 National Institute of Clinical Excellence (NICE) guideline we aimed to identify neonates at risk of sepsis and initiate treatment early. The purpose of the audit was to assess Craigavon Area Hospital's adherence to this guideline.

Methodology All neonates who were commenced on antibiotics within the first $72 \mathrm{~h}$ of life between 9th-28th September 2013 were identified. A re-audit was carried out between 5th-19th January 2014. 17 patients were included in the first audit and 15 in the second cycle. Clinical notes were retrospectively analysed by two investigators. The NICE audit tool was used to examine domains including; time taken to commence treatment, use of the correct dose regime of appropriate antibiotics, appropriate blood tests and prompt action on results. As a result of the first audit cycle a checklist was developed highlighting risk factors for sepsis and NICE guidelines. This was included in medical notes as a prompt for junior doctors. The re-audit evaluated for improved performance.

Results and conclusions Overall the unit adhered well to the guideline. Areas for improvement included; availability of culture results within $36 \mathrm{~h}$ and repeat of inflammatory markers 18-24 h after commencing antibiotics. Introduction of the checklist improved observation of NICE guidelines overall although availability of culture results remained problematic.

\section{PO-0552 CHANGING EPIDEMIOLOGY OF STAPHYLOCOCCUS AUREUS IN A TERTIARY NEONATAL INTENSIVE CARE UNIT (NICU), 2008-2013}

${ }^{1} \mathrm{~L}$ McKechnie, ${ }^{1} \mathrm{~S} J$ English, ${ }^{2} \mathrm{~K}$ Sethi. ${ }^{1}$ Department of Neonatology, Leeds Teaching Hospital NHS Trust, Leeds West Yorkshire, UK; ${ }^{2}$ Department of Microbiology, Leeds Teaching Hospital NHS Trust, Leeds West Yorkshire, UK

\subsection{6/archdischild-2014-307384.1194}

Background and aims S.aureus is the second most common pathogen causing late onset septicemia in NICUs, particularly in premature infants with very low birth weight. Poorly developed host defence mechanisms, the necessity for central venous catheters, invasive procedures, poor skin integrity, prolonged total parenteral nutrition and the use of steroids or antimicrobial agents all increase the risk of staphylococcal infection in premature infants. 